\title{
Perfil sociodemográfico asociado a la ideación suicida de las personas transexuales.
}

\section{Sociodemographic profile associated with the suicidal ideation of transsexual people.}

Fecha de recepción: 24-07-2020

Fecha de aceptación: 16-11-2020

\author{
María Dolores Gil-Llario \\ Catedrática de Universidad. \\ Departamento de Psicología Evolutiva y de la Educación. \\ Facultad de Psicología. Universitat de València \\ Olga Angustias Fernández-García \\ Personal Investigador. Dpto. Psicología Evolutiva y de la Educación. \\ Facultad de Psicología. Universitat de València \\ Trinidad Bergero Miguel \\ Psicóloga de la Unidad de Identidad de Género del \\ Hospital Carlos Haya de Málaga
}

\section{resumen/alsstract:}

Las personas transexuales experimentan tasas extremadamente altas de conductas suicidas. Sin embargo, aunque la ideación suicida se considera un precursor importante de intento de suicidio, se ha prestado poca atención a esta problemática y al perfil sociodemográfico de este colectivo. Así, nos proponemos identificar la prevalencia de ideación suicida en una muestra de pacientes transexuales y explorar su perfil sociodemográfico. Para ello, se recogió información sociodemográfica de 172 transexuales (58.7\% mujeres transexuales, $41.3 \%$ hombres transexuales) de entre 18 y 61 años que acudieron a la Unidad de Trastornos de Identidad de Género del Complejo Hospitalario Carlos Haya. Los resultados revelan una tasa de ideación suicida del $61 \%$ y en el $56.2 \%$ de los casos la ideación suicida actuó como precursor de intentos de suicidio. Los pacientes con ideación suicida parecen presentar un amplio historial en salud mental, aunque menos ingresos psiquiátricos y un menor consumo de psicofármacos, pero no de cocaína y marihuana. Además, la pérdida de amigos/as, la expulsión del hogar, las autolesiones no suicidas y/o la victimización por razón de género también se identificaron como factores precursores. Así, queda evidenciada la necesidad de elaborar programas de prevención e intervención que se ajusten a las características de este colectivo.

Transgender people experience extremely high rates of suicidal behavior. However, although suicidal ideation is considered an important precursor to suicide attempts, little attention has been paid to this problem and to the sociodemographic profile of this group. Thus, we propose to identify the prevalence of suicidal ideation in a sample of transsexual patients and to explore their sociodemographic profile. To this end, sociodemographic information was collected from 172 transsexuals (58.7\% female transsexuals, $41.3 \%$ male transsexuals) between the ages of 18 and 61 who attended the Gender Identity Disorder Unit at the Carlos Haya Hospital Complex. The results revealed a 61\% rate of suicidal ideation, and in $56.2 \%$ of cases, suicidal ideation acted as a precursor to suicide attempts. Patients with suicidal ideation appear to have an extensive mental health history, although fewer psychiatric admissions and less use of psychotropic drugs, but not cocaine and marijuana. In addition, loss of friends, removal from the home, non-suicidal self-harm and/or gender-based victimization were also identified as precursor factors. Thus, the need to develop prevention and intervention programmes that fit the characteristics of this group is evident.

\section{palahras clave/keywords:}

Ideación suicida; Transexualidad; Perfil sociodemográfico

Suicidal ideation; Transsexuality; Sociodemographic profile

María Dolores Gil-Llario. Facultad de Psicología. Universitat de València. España. Correspondencia: dolores.gil@uv.es 


\section{Introducción}

El suicidio se considera un problema de salud pública global ya que casi 1 millón de personas fallecen por este motivo cada año en todo el mundo (OMS, 2019). En España, los últimos datos oficiales indican que 3.539 personas fallecieron por suicidio (2.619 hombres y 920 mujeres) en 2018 (INE, 2019), situándose como la primera causa externa de defunción en nuestro país, desde hace más de una década. Sin embargo, las iniciativas para su prevención son escasas o nulas, lo que ha llevado a la Confederación de SALUD MENTAL ESPAÑA a reivindicar la elaboración de un Plan Nacional para la Prevención del Suicidio, con el propósito de paliar un problema de salud pública más agravado si cabe en los últimos meses con la pandemia del COVID-19 (Confederación SALUD MENTAL ESPAÑA, 2020).

Entre los grupos con mayor riesgo de experimentar conductas suicidas, las personas lesbianas, gays, bisexuales y transexuales (LGBT) han sido las que han mostrado una de las prevalencias más elevadas (Claes et al., 2015; Haas et al., 2011; Tomicic et al., 2016). Cano et al. (2004) en su estudio con 200 pacientes con diagnóstico clínico de transexualidad que eran atendidos en la UTIG de Málaga, expusieron que 59.3\% mujeres transexuales y $40.7 \%$ hombres transexuales decían haber presentado ideación suicida a lo largo de su vida, y en el $29.6 \%$ y $30.6 \%$ de las ocasiones, respectivamente, se había consumado un intento de suicidio. Estudios recientes como el de Modrego et al. (2020), quienes examinaron la prevalencia de conductas suicidas en 190 adultos jóvenes transexuales atendidos en la UIG de la Comunidad Valenciana, obtuvieron datos semejantes ya que el 50\% había presentado ideación suicida, aunque el número de personas que llegaron a realizar los actos suicidas bajó hasta el $21 \%$. Esta asociación ha sido explicada por el modelo de estrés en minorías, que plantea que pertenecer a una minoría discriminada deja expuesto al individuo a un entorno social hostil caracterizado por el prejuicio, el rechazo y la exclusión (Meyer, 2003).

En concreto, las personas transexuales presentan una identidad o expresión de género que es diferente del sexo asignado al nacer, y aunque no siempre, en ocasiones esa fuerte y persistente identificación con el sexo opuesto e incomodidad con el propio sexo supone un desafío vital que conlleva angustia psicológica y sintomatología depresiva (Claes et al., 2015). Esto acrecienta la probabilidad de que este colectivo presente problemas de salud física y psicológica, y se asocia con un mayor riesgo de intento de suicidio (Marshall, Claes, Bouman, Witcomb y Arcelus, 2016). A pesar de ello, se trata de un grupo social que ha recibido una atención limitada (Haas et al., 2011), y, por tanto, se desconoce cuáles son los precursores de los intentos de suicidios en las personas transexuales, lo que dificulta la intervención frente a esta problemática (Liu y Mustanski, 2012).

En esta línea, un precursor importante de los intentos de suicidio es la ideación suicida. Según estudios recientes, aproximadamente en el 29\% de los casos la ideación suicida evoluciona hacia un intento de suicidio, siendo especialmente alto el riesgo durante el primer año de inicio de la ideación (Nock et al., 2008a). Además, el inicio de la ideación suicida suele producirse alrededor de los 12 años, alcanzando el momento de mayor riesgo durante la adolescencia media y tardía (Liu y Mustanski, 2012; Nock et al., 2008b). Así, es importante destacar que la ideación y los intentos de suicidio presentan vías causales distintas (Wol- 
ford-Clevenger, Frantell, Smith, Flores y Stuart, 2018), y, por tanto, conocer los factores asociados a la ideación suicida será esencial para comprender mejor su etiología y prevenir este problema de salud mental (Liu y Mustanski, 2012).

Respecto al perfil sociodemográfico de las personas transexuales con ideación suicida, se ha encontrado que suelen ser adolescentes o adultos jóvenes con sexo biológico masculino, un bajo nivel educativo y económico, y sin pareja sentimental (Chen et al., 2019; Haans et al., 2011; Marshall et al., 2016). Además, los problemas de salud mental, particularmente la depresión, también han sido identificados como factores precursores de la ideación suicida, dada la asociación que se ha encontrado entre asistir a consultas de salud mental y tomar medicación psiquiátrica en los pacientes con la ideación suicida. Pero también hay estudios que han incidido en los antecedentes de abuso de sustancias y abuso físico, psicológico o sexual como factores precursores de la ideación suicida (Chen et al., 2019; Marshall et al., 2016). En esta línea, se ha encontrado una relación positiva y significativa entre las experiencias de discriminación y victimización por razón de género y las tasas de ideación suicida (Testa et al., 2017). Así mismo, la prevalencia de autolesiones no suicidas parece ser un correlato importante de la ideación suicida (Aboussouan, Snowb, Cerelb y Tuckera, 2019), ya que en el estudio de Bryan y Bryan (2014) el $86.7 \%$ de los participantes que informaron haber participado en autolesiones no suicidas presentaba ideas de suicidio.

A pesar de todo lo expuesto anteriormente y de la evidente vulnerabilidad de este colectivo a experimentar conductas suicidas, se carece de conclusiones representativas acerca de la prevalencia de la ideación suicida en personas transexuales. Esto es debido, por un lado, a la focalización de la atención en los intentos de suicidio y no en sus factores precursores (p.ej. intentos de suicidio), y, por otro lado, a la dificultad para acceder a la muestra, lo que complica la obtención de datos generalizables (Eisenberg et al., 2017; Liu y Mustanski, 2012). Así, actualmente existe una evidente escasez de literatura sobre la prevención del suicidio en este colectivo que dificulta el desarrollo de intervenciones dirigidas a este grupo poblacional específico y que está ocasionando un incremento de los problemas de salud mental (Moody y Smith, 2013). El objetivo de este estudio, por tanto, es investigar la tasa de ideación suicida en una muestra de pacientes transexuales y, recopilar y analizar información sobre su perfil sociodemográfico con el propósito de identificar posibles factores asociados a estos pensamientos.

\section{Material y métodos \\ Participantes}

La muestra del presente estudio está constituida por 172 personas transexuales que acudieron a la Unidad de Trastornos de Identidad de Género (UTIG) del Complejo Hospitalario Carlos Haya de Málaga. El 58.7\% de los participantes $(n=101)$ son mujeres transexuales mientras que el $41.3 \%(n=71)$ hombres transexuales, con un rango de edad de entre los $18 \mathrm{y}$ los 61 años $(M=29.21, D T=8.71)$. En cuanto al estado civil, la mayoría son solteros $(76.9 \%)$ o conviven con su pareja (20.9\%). Por lo que respecta a su ocupación, el $49.7 \%$ tiene un 
trabajo remunerado a tiempo completo o parcial, un $12.6 \%$ está en situación de desempleo y un $13.2 \%$ es estudiante.

Los criterios de inclusión fueron: tener un diagnóstico de Transexualismo en la UTIG, no haber sido sometido previamente a ningún tipo de intervención médica o quirúrgica, ser mayor de edad y tener la suficiente competencia en lecto-escritura como para cumplimentar los instrumentos. El único criterio de exclusión fue presentar un diagnóstico de psicosis o trastornos de personalidad graves.

\section{Instrumentos}

Cuestionario de Información Sociodemográfica de la Unidad de Trastornos de Identidad de Género (UTIG) (Bergero y Cano, 2006). Este instrumento forma parte de un protocolo de evaluación más amplio compuesto por entrevistas estructuradas e instrumentos diagnósticos. Consta de un total de 79 ítems de formato variado con preguntas de respuesta dicotómica, de elección múltiple y también algunas abiertas, y permite recabar información sobre distintos ámbitos personales e interpersonales considerados de gran interés en personas transexuales. Concretamente, se centra en la exploración de variables sociodemográficas y psicológicas de interés, comorbilidad psiquiátrica, antecedentes familiares y personales de salud mental, conductas autolíticas, eventos estresantes, apoyo social y familiar, y uso de fármacos y drogas. Para el presente estudio únicamente se han utilizado 48 ítems. Un ítem recoge información sobre la edad, otro sobre el sexo biológico (hombre y mujer) y otro sobre el estado civil (soltero/a, convive con la pareja, soltero/a, divorciado/a, separado/a y viudo/a) de los encuestados. Ocho ítems hacen referencia a los antecedentes personales de salud mental: alguna vez has acudido a una consulta de salud mental (sí/no), alguna vez has recibido tratamiento de salud mental (sí/no), qué tipo de tratamiento (psicoterapia, farmacoterapia o ambas), alguna vez has tomado medicación para la depresión, para dormir o para la ansiedad (sí/no), has sufrido algún ingreso en la unidad psiquiátrica (sí/no) y cuál fue el motivo (respuesta abierta). Un ítem pregunta sobre la presencia de ideas de automutilación (sí/no), y veintidós ítems con formato de respuesta dicotómica (sí/no) exploran la vivencia de eventos estresantes: muerte de un familiar, de la madre, del padre, de la pareja, de un hermano o de un amigo/a, enfermedad grave de los padres o de uno mismo, cuidado de un enfermo crónico, separación o divorcio de los padres, perdida del trabajo o de la casa, detenciones, ingresos en la cárcel o en el servicio militar o experiencia de agresión física, psicológica o sexual. Respecto a este último aspecto se recoge información no solo sobre el tipo de agresión (física, psicológica o sexual), sino también sobre el agresor (respuesta abierta), si la considera grave (sí/no), y si necesitó atención médica u hospitalaria (sí/no). Ocho ítems con formato de respuesta dicotómica (sí/no) exploran el consumo de las siguientes drogas: marihuana, cocaína, heroína, otros opiáceos, alucinógenos, anfetaminas, drogas de diseño e inhalantes. Y seis ítems recogen información sobre la variable criterio: has tenido ideas suicidas (sí/no), edad de la primera ideación suicida (respuesta abierta), tiempo que se han mantenido (respuesta abierta), has llevado a cabo algún intento de suicidio (sí/no), número de intentos de suicidio (respuesta abierta), edad a la que tuvo lugar el intento de suicidio (respuesta abierta). Para el presente estudio se ha requerido categorizar las variables de respuesta abierta. 


\section{Procedimiento}

Los participantes mayores de edad diagnosticados de Transexualismo fueron reclutados de manera sucesiva en la UTIG del Hospital Universitario Carlos Haya de Málaga entre los años 2001-2008.

Durante el proceso de evaluación diagnóstica, la psicóloga clínica -previo consentimiento- era la encargada de administrar la batería de instrumentos. El protocolo de evaluación formado por el cuestionario de información sociodemográfica ya indicado y por el Cuestionario de Comportamiento Sexual para Transexuales de Málaga (Bergero, Ballester, Gornemann, Cano y Asiain, 2010) permitía recabar información necesaria para el establecimiento del diagnóstico y la toma de decisiones futuras. Por tanto, todo el proceso de evaluación objeto de estudio en este trabajo fue previo a cualquier intervención endocrinológica o quirúrgica. La heteroadministración de la batería requería unas cuatro horas aproximadamente.

En primer lugar, aplicando los criterios de exclusión, se descartaron personas con diagnóstico de psicosis o trastornos de personalidad graves (HBIGDA, 1998). Para el diagnóstico, se siguieron los criterios CIE-10 y en todo el proceso de evaluación, las recomendaciones de los Estándares Asistenciales de The World Professional Association for Transgender Health (HBIGDA, 1998, 2001).

Todos los participantes fueron informados del objetivo del estudio y dieron su consentimiento para participar en el mismo sabiendo que podían abandonar la investigación en el momento en que quisieran.

\section{Análisis de datos}

Se emplearon estadísticos descriptivos, tablas de frecuencias y de contingencia para la obtención de la tasa de prevalencia de ideación suicida del grupo muestral, así como para el análisis de su distribución en función de las diferentes variables sociodemográficas estudiadas. Por otro lado, la prueba Chi-cuadrado y prueba exacta de Fisher nos permitieron estimar el grado de asociación entre las diferentes variables cualitativas examinadas, y la correlación de Pearson entre las variables cuantitativas. Todos los análisis estadísticos se realizaron con el programa IBM SPSS Statistics 23.

\section{Resultados}

\section{Prevalencia de ideación suicida en personas transexuales}

La prevalencia de ideación suicida autoinformada en esta muestra de personas transexuales es del $61 \%(n=105)$. Respecto a la edad de la primera ideación suicida, el $43.3 \%$ manifiesta haberla experimentado antes de los 15 años, el $40.4 \%$ entre los 16 y 22 años, el $11.5 \%$ entre los 23 y 29 años, y únicamente el $4.8 \%$ después de cumplir los 30 años. Ahora bien, si analizamos la relación entre la edad de la primera ideación suicida y el tiempo que estas se han mantenido, encontramos que el $61 \%$ de quienes tuvieron las primeras ideas suicidas antes de los 15 años manifiestan que estas perduraron entre 7 y 29 años, mientras que el $80 \%$ de quienes las experimentaron por primera vez entre los 30 y los 37 años tuvieron estos pensamientos durante un máximo de 6 años (Figura 1). La relación entre estas dos variables es negativa y estadísticamente significativa $(\mathrm{r}=-.34, \mathrm{p}<.001)$. 
Figura 1. Distribución de los participantes según la edad de inicio de la primera ideación suicida y el tiempo que estas se han mantenido.

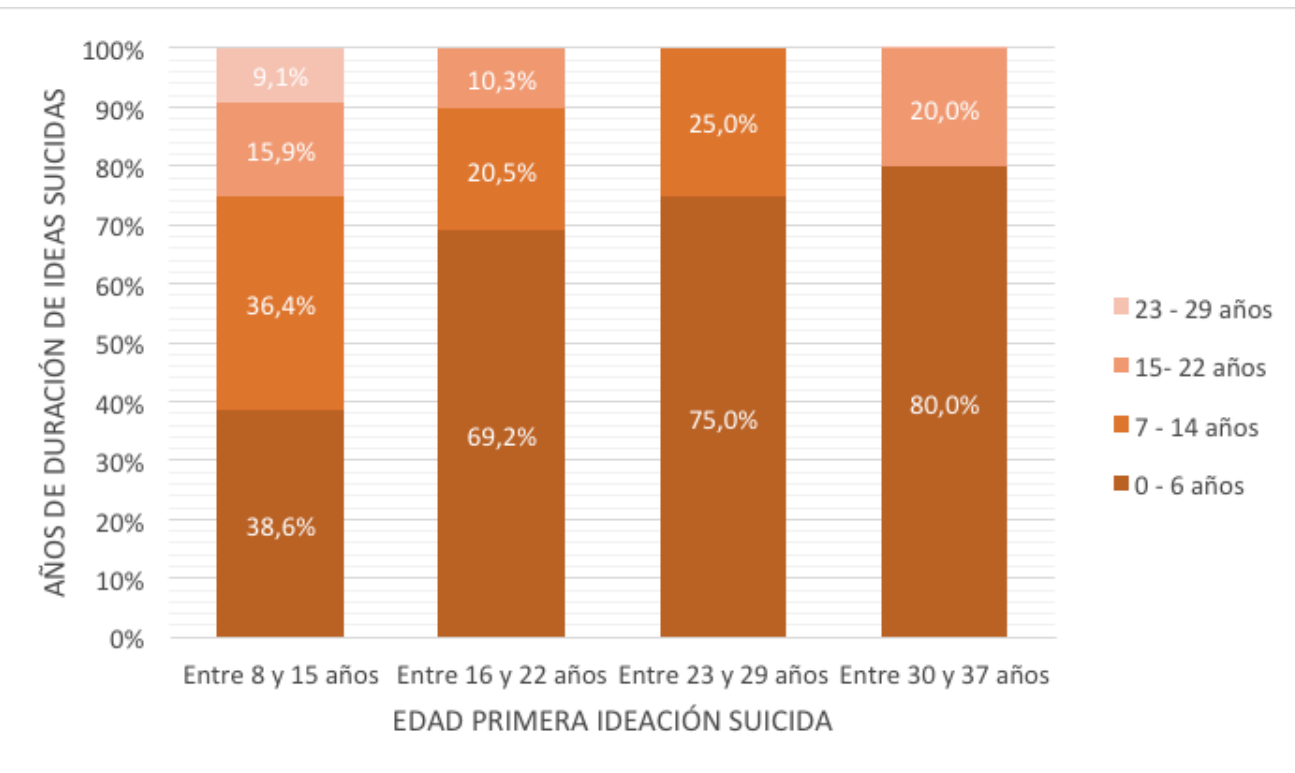

Figura 2. Distribución de los participantes en función de su edad cronológica y la edad de inicio de la primera ideación suicida.

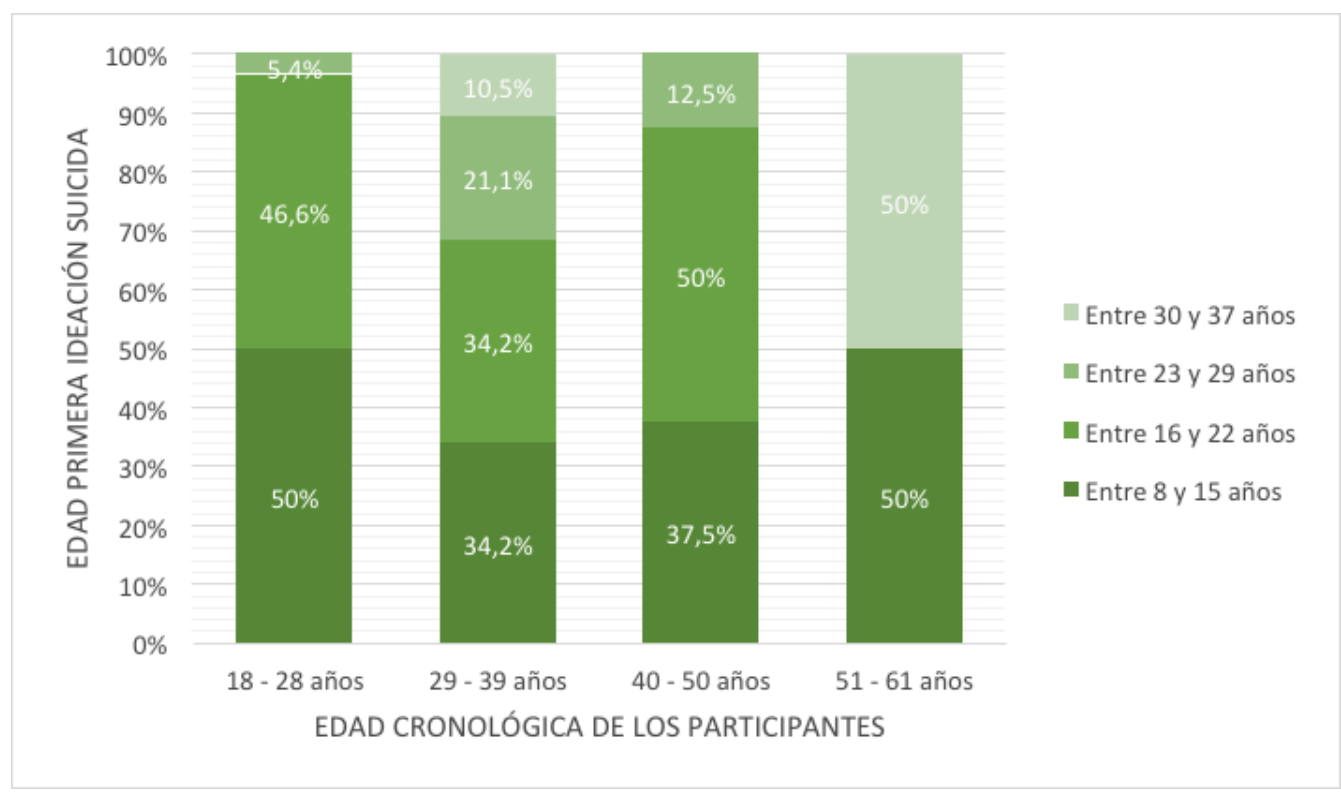


Por otro lado, la relación entre la edad cronológica de los participantes y la edad de la primera ideación suicida sí se ha mostrado positiva y estadísticamente significativa $(\mathrm{r}=.33, \mathrm{p}$ $=.001$ ), ya que el $94.6 \%$ de los participantes más jóvenes (entre 18 y 28 años) afirma haber experimentado las primeras ideas suicidas antes de los 22 años, mientras el 50\% de los participantes mayores de 51 años experimentó la primera ideación suicida después de los 30 años (Figura 2).

Así mismo, el 56.2\% de los participantes que ha experimentado ideación suicida en algún momento de su vida declara haber realizado algún intento de suicidio. La relación entre ambas variables (ideación suicida e intentos de suicidio) se muestra positiva y estadísticamente significativa $\left(\chi^{2}=57.3, \mathrm{p}<.001\right)$. Además, entre aquellos que experimentaron ideación suicida, el $67.8 \%$ ha llevado a cabo entre uno y dos intentos de suicidio mientras el $27.3 \%$ tuvo tres o más. Así mismo, se ha encontrado que el $49.2 \%$ de quienes consumaron un intento de suicidio experimentaron la primera ideación suicida entre los 8 y los 15 años, el 37.3\% entre los 16 y los 22 años, el $8.5 \%$ entre los 23 y los 29 años, y solo el $5.1 \%$ entre los 30 y los 37 años. En cambio, entre aquellos que tuvieron ideación suicida pero no han realizado ningún intento de suicidio, las primeras ideas de suicidio aparecen a edades más tardías (Tabla 1). A pesar de ello, ambas variables (intento de suicidio y edad de la primera ideación suicida) no han mostrado una relación estadísticamente significativa $\left(\chi^{2}=2.54, \mathrm{p}=.467\right)$. Sin embargo, sí se ha encontrado una relación positiva y estadísticamente significativa $(\mathrm{r}=.608, \mathrm{p}<.001)$ entre edad de la primera ideación suicida y la edad del intento de suicidio.

Tabla 1. Frecuencia de participantes en los rangos de edad de la primera ideación suicida en función de si realizaron o no intento de suicidio.

Edad de la primera ideación suicida

\begin{tabular}{cccccc} 
& & $\begin{array}{c}\text { Entre 8 y 15 } \\
\text { años }\end{array}$ & $\begin{array}{c}\text { Entre 16 y 22 } \\
\text { años }\end{array}$ & $\begin{array}{c}\text { Entre 23 y } \\
29 \text { años }\end{array}$ & $\begin{array}{c}\text { Entre 30 y 37 } \\
\text { años }\end{array}$ \\
\hline \multirow{2}{*}{ Intento de suicidio } & Sí & $49.2 \%$ & $37.3 \%$ & $8.5 \%$ & $5.1 \%$ \\
& No & $35.6 \%$ & $44.4 \%$ & $15.6 \%$ & $4.4 \%$ \\
\hline
\end{tabular}

\section{Características sociodemográficas de los pacientes con ideación suicida}

Si analizamos la distribución de la muestra en función de las variables sociodemográficas, encontramos que las mujeres transexuales experimentan ideas suicidas con mayor frecuencia $(57.1 \%)$ que los hombres transexuales $(42.9 \%)$, y que la mayoría de los participantes con ideación suicida tienen entre 18 y 28 años (53.3\%) y son solteros/as (77.1\%) (Tabla 2). A pesar de ello, ninguna de estas variables sociodemográficas muestra una relación estadísticamente significativa con la ideación suicida.

Respecto a los antecedentes personales de salud mental (SM) (Figura 3), el 78.1\% de los participantes con ideación suicida ha acudido alguna vez a la consulta de SM y el 62.9\% ha recibido tratamiento de SM en alguna ocasión, detectándose diferencias estadísticamente significativas entre los grupos muestrales (pacientes con y sin ideación suicida) $\left(\chi^{2}=13.93\right.$, 
Tabla 2. Distribución de los pacientes con ideación suicida en función de las variables sexo biológico, edad y estado civil.

\begin{tabular}{|c|c|c|c|c|c|c|c|c|c|c|c|c|c|}
\hline & \multicolumn{3}{|c|}{ Sexo biológico } & \multicolumn{4}{|c|}{ Grupos de edad } & \multicolumn{6}{|c|}{ Estado civil } \\
\hline & Hombre & Mujer & $\chi^{2}$ & $\begin{array}{c}18-28 \\
\text { años }\end{array}$ & $\begin{array}{l}29-39 \\
\text { años }\end{array}$ & $\begin{array}{c}40-50 \\
\text { años }\end{array}$ & $\begin{array}{c}51-61 \\
\text { años }\end{array}$ & $\chi^{2}$ & Casado/a & $\begin{array}{c}\text { Convive } \\
\text { con la } \\
\text { pareja }\end{array}$ & Soltero/a & Separado/a & $\chi^{2}$ \\
\hline $\begin{array}{c}\text { Ideación } \\
\text { Suicida }\end{array}$ & $57.1 \%$ & $42.9 \%$ & 1.86 & $53.3 \%$ & $36.2 \%$ & $7.6 \%$ & $2.9 \%$ & 0.27 & $0 \%$ & $21.9 \%$ & $77.1 \%$ & $1 \%$ & 2.16 \\
\hline
\end{tabular}

$\mathrm{p}<.001 ; \chi^{2}=17.82, \mathrm{p}<.001$, respectivamente). En el caso de los participantes que han sido tratados en SM, la mayoría ha recibido psicoterapia (42.4\%), frente al $28.8 \%$ que ha recibido farmacoterapia y el resto que se ha beneficiado de ambos tratamientos. En relación a la toma de medicamentos para el tratamiento de la depresión, ansiedad e insomnio, los análisis muestran que un bajo porcentaje de participantes con ideación suicida ha tomado este tipo de fármacos en alguna ocasión $\left(\chi^{2}=11.22, \mathrm{p}=.001 ; \chi^{2}=8.21, \mathrm{p}=.016 ; \chi^{2}=9.59\right.$, $\mathrm{p}$ $=.022$, respectivamente). Por otro lado, la mayoría de las personas que han experimentado ideas suicidas manifiesta no haber sufrido ningún ingreso en la unidad psiquiátrica (89.5\%) $\left(\chi^{2}=7.5, p=.007\right)$. El $10.5 \%$ que sí lo hizo, declara que el ingreso estuvo motivado por un intento autolítico.

Figura 3. Antecedentes personales de salud mental en pacientes con ideación suicida

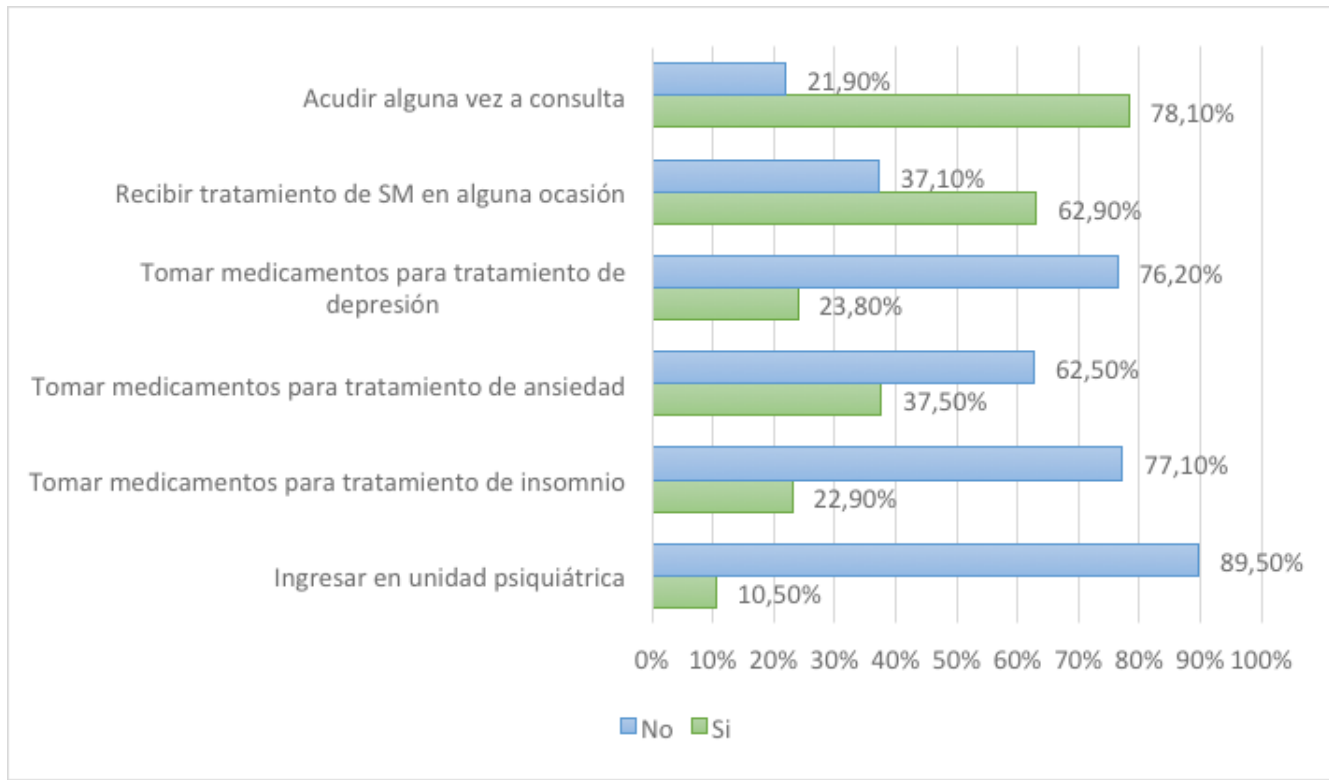


En esta línea, los resultados indican que los participantes que han experimentado ideas de automutilación son significativamente más propensos a informar de ideas de suicidio $(85.7 \%)\left(\chi^{2}=21.66, \mathrm{p}<.001\right)$.

En el caso del uso de drogas, aquellos participantes que han consumido marihuana (74.6\%) o cocaína (84.4\%) en alguna ocasión, son significativamente más propensos a informar de ideación suicida $\left(\chi^{2}=8.51, \mathrm{p}=.004 ; \chi^{2}=8.99, \mathrm{p}=.002\right.$, respectivamente). No encontramos asociación estadísticamente significativa entre la ideación suicida y el consumo de otras drogas (otros opiáceos, $\chi^{2}=1.38, \mathrm{p}=.239$; alucinógenos, $\chi^{2}=2.75, \mathrm{p}=.097$; anfetaminas, $\chi^{2}=1.48, \mathrm{p}=.224$; drogas de diseño, $\chi^{2}=1.8, \mathrm{p}=.179$; inhalantes, $\chi^{2}=.68, \mathrm{p}=.407$ ).

De acuerdo a los eventos vitales estresantes, se ha encontrado que tanto aquellos participantes que han sido expulsados de su hogar (80\%) como aquellos que han sufrido la pérdida de un amigo (77.4\%), se muestran significativamente más propensos a experimentar episodios de ideación suicida que aquellos que no han vivenciado estos episodios $\left(\chi^{2}=3.9, \mathrm{p}=.048\right.$; $\chi^{2}=5, \mathrm{p}=.026$, respectivamente).

Por último, respecto a la vivencia de discriminación y victimización por razón de género, el $77.67 \%$ de los pacientes con ideación suicida han sido maltratados o agredidos en alguna ocasión $\left(\chi^{2}=18.35, \mathrm{p}<.001\right)$. Afirman que en el $34.6 \%$ de las ocasiones han sufrido estas agresiones durante toda la vida, tratándose de vejaciones verbales o emocionales en el $48.1 \%$ de los casos y siendo ejercidas en la mayoría de las ocasiones por parte de un desconocido (28.4\%) o de su padre (22.2\%). Sin embargo, casi todos las califican como leves $(88.6 \%)$ y no necesitaron asistencia médica $(92.5 \%)$ ni hospitalaria $(93.8 \%)$.

\section{Discusión}

Este estudio tenía el objetivo de analizar la prevalencia de ideación suicida en una muestra de individuos transexuales, así como explorar el perfil sociodemográfico de estos pacientes.

Respecto al primer objetivo, los resultados de este estudio permiten confirmar la alta prevalencia de ideación suicida en las personas transexuales, ya que el $61 \%$ de la muestra afirmó haber presentado este tipo de pensamientos en alguna ocasión. Estos datos van en la línea de lo publicado en recientes estudios sobre este colectivo. Así, Chen et al. (2019) encontraron una prevalencia de ideación suicida del $56.4 \%$ en una muestra de individuos transexuales chinos y Eisenberg et al. (2017) del $61.3 \%$ en una muestra de pacientes de la UIG en el Reino Unido. Fue menor la hallada por Liu y Mustanski (2012) (37.4\%) y Perez-Brumer, Day, Russell y Hatzenbuehler (2017) (35\%), quienes exploraron los comportamientos suicidas de jóvenes menores de 20 años de Chicago y estudiantes universitarios de California, respectivamente. Estas prevalencias más bajas podrían ser explicadas por las diferencias respecto a la edad de las muestras en las que se basan estas dos últimas investigaciones. Así mismo, de acuerdo con lo planteado en la investigación de Nock et al. (2008b) de Estados Unidos, más de la mitad de los participantes de nuestro estudio que referencian haber presentado una idea suicida en alguna ocasión declaran haberlo hecho por primera vez antes de los 16 años. Además, se ha encontrado que quienes tuvieron las primeras ideas suicidas a edades más tempranas las experimentaron durante periodos más largos de tiempo y que los participantes más jóvenes han tenido ideas suicidas a edades más tempranas. 
Por otro lado, los resultados de nuestro estudio confirman que la ideación suicida actúa como factor precursor de los intentos de suicidio, ya que el 56.2\% de los participantes con ideas suicidas llevaron a cabo intentos suicidas. Estos resultados van en la misma línea que lo planteado por Nock et al. (2008b) en su investigación con pacientes estadounidenses y Terada et al. (2011) en su estudio con pacientes japoneses, quienes encontraron que los intentos de suicidio son más frecuentes entre aquellos que presentan ideas suicidas (29\% y $43.3 \%$, respectivamente). En la mayoría de las ocasiones, los participantes con ideas suicidas previas realizaron menos de 3 intentos de suicidio. Además, a medida que se atrasa la edad de la primera ideación suicida disminuye la probabilidad de realizar intentos de suicidio ya que tal y como declaran Nock et al. (2008a) en su estudio, el riesgo de intento de suicidio es especialmente alto en aquellas personas que han experimentado ideas de suicidio a una edad más temprana. Esto podría deberse a que, posiblemente, estas ideas se instauran de forma más arraigada a estas edades, causando mayor impacto y peores consecuencias.

Respecto al segundo objetivo de nuestro estudio, no se encontró una asociación estadísticamente significativa entre el sexo biológico y la ideación suicida, aunque fueron las mujeres transexuales quienes presentaron más ideas suicidas. Esto va en la línea de lo establecido en la revisión bibliográfica de Marshall et al. (2016) y podría explicarse por la tendencia de las personas de género femenino a reportar más problemas de salud mental. Además, también encontramos que es más frecuente la ideación suicida entre pacientes más jóvenes y entre aquellos que no tenían pareja sentimental, lo que coincide con lo postulado en otros estudios (Chen et al., 2019; Haans et al., 2011; Marshall et al., 2016). Estos resultados podrían ser explicados por lo postulado por algunos autores que han demostrado que el apoyo social actúa como factor protector contra el suicidio (Eisenberg et al., 2017). A pesar de ello, no se ha encontrado relación entre la tasa de prevalencia de ideación suicida y las variables grupos de edad, estado civil, nivel de estudios o situación laboral, tal y como ocurre en el estudio de Terada et al. (2011) con pacientes japoneses, una muestra con características parecidas a la de nuestro estudio.

Así mismo, la ideación suicida también parece estar asociada a los problemas de salud mental, ya que encontramos una mayor frecuencia de pacientes de salud mental entre aquellas personas que referían haber tenido ideas suicidas. Estos resultados concuerdan con lo postulado por Chen et al. (2019) y Marshall et al. (2016), con muestras semejantes a la de nuestro estudio, quienes afirman que las personas transexuales con ideación suicida hacen un mayor uso de los servicios de salud mental. Estos resultados podrían explicarse por la alta prevalencia de problemas de salud mental en personas transexuales dada la angustia e incomodidad que experimentar. A pesar de ello, los resultados de este estudio informan de un menor consumo de psicofármacos y menos ingresos psiquiátricos entre los pacientes con ideación suicida. Esto podría estar relacionado con el miedo a la discriminación que persigue a este colectivo en los diferentes ámbitos, y sobre todo en el sanitario (Aboussouan et al., 2019).

Por otro lado, la mayoría de los participantes de nuestra muestra que presentó conductas autolíticas no suicidas informaron haber tenido en alguna ocasión ideas de suicidio, algo 
que concuerda con lo encontrado por Aboussouan et al. (2019) en su estudio con veteranos de guerra de Estados Unidos.

El consumo de marihuana o cocaína en algún momento de la vida también se ha relacionado con la ideación suicida. Tal y como afirma Mathy (2002), citado por Moody y Smith (2013), los participantes que presentan un consumo de alcohol o drogas previo informan de mayor ideación suicida que aquellos que nunca han consumido, posiblemente porque la drogodependencia aumenta significativamente el riesgo de padecer trastornos depresivos que pueden culminar con el suicidio.

Entre los eventos vitales estresores analizados, la pérdida de un/a amigo/a y la expulsión del hogar parecen actuar como factores precursores de las ideas de suicidio en nuestro estudio. De acuerdo con lo postulado por Lehavot, Simpson y Shipherd (2016) y Marshall et al. (2016) esta asociación positiva podría ser el resultado de experiencias de estrés de minorías como son la perdida de apoyos sociales que actúan como atenuantes del riesgo de suicidio y el rechazo o discriminación en el acceso a una vivienda estable.

En relación con esto último, gran parte de los pacientes con ideación suicida de nuestro estudio sostiene haber sufrido discriminación o haber sido agredidos por razón de género en alguna ocasión. Parece tratarse de algo habitual entre las personas transexuales que experimentan estos pensamientos ya que varios estudios han corroborado esta asociación (Claes et al., 2015; Liu y Mustanski, 2012; Marshall et al., 2016; Perez-Brumer et al., 2017; Taliaferro, McMorris y Eisenberg, 2018; Wolford-Clevenger et al., 2018).

Considerando todo lo expuesto, podríamos afirmar que esta investigación no solo contribuye a mejorar el conocimiento científico relativo a la conducta suicida en los individuos transexuales, sino que también aporta información muy valiosa para la elaboración de programas de prevención e intervención más ajustados a las características de este colectivo. Concretamente, en nuestro país, son muchos los profesionales de salud mental que están demandando la implantación de una estrategia nacional de prevención del suicidio que abarque desde la formación a profesionales de la salud hasta la elaboración de campañas de sensibilización social y visibilización del problema. En esta línea, este estudio aporta información muy valiosa sobre un colectivo que presenta mayor incidencia, lo que puede contribuir a la elaboración de medidas más eficaces y de mayor impacto social. Así, cabe resaltar la importancia de disminuir la estigmatización social que rodea a este colectivo, sobre todo entre los profesionales de salud mental ya que la atención recibida en este ámbito es primordial para paliar este problema. La elaboración de campañas de sensibilización dirigidas al personal sanitario que hicieran hincapié en la posible vulnerabilidad de este colectivo podría ayudar en esta labor.

Por último, futuras investigaciones deberían contrastar los resultados obtenidos en una muestra no clínica de personas transexuales, ya que en nuestro estudio los pacientes habían acudido a la Unidad de Trastornos de Identidad de Género lo que sería un indicador de mayor malestar y angustia. 


\section{Referencias bibliográficas}

Aboussouan, A.; Snow, A.; Cerel, J.; Tucker, R.P. (2019). Non-suicidal self-injury, suicide ideation, and past suicide attempts: comparison between transgender and gender diverse veterans and non-veterans. Journal of affective disorders, 259, 186-194. https://doi.org/10.1016/j.jad.2019.08.046

Bergero, T.; Cano, G. (2006). El proceso diagnóstico. En E. Gómez e I. Esteva (Eds.), Ser transexual. Barcelona: Ed. Glosa.

Bergero, T.; Ballester, R.; Gornemann, I.; Cano, G.; Asiain, S. (2012). Desarrollo y validación de un instrumento para la evaluación del comportamiento sexual de los transexuales: el CSTM. Revista de Psicopatología y Psicología Clínica, 17(1), 11-30.

Bryan, C.J.; Bryan, A.O. (2014). Nonsuicidal self-injury among a sample of united states military personnel and veterans enrolled in college classes. J. Clin. Psychol. 70, 874-885. https://doi.org/10.1002/jclp.22075

Cano Oncala, G.; Bergero Miguel, T.; Esteva de Antonio, I.; Giraldo Ansio, F.; Gómez Banovio, M.; Gorneman Schaffer, I. (2004). La construcción de la identidad de género en pacientes transexuales. Revista de la Asociación Española de Neuropsiquiatría, (89), 21-30.

Chen, R.; Zhu, X.; Wright, L.; Drescher, J.; Gao, Y.; Wu, L.; ... Broome, M. (2019). Suicidal ideation and attempted suicide amongst Chinese transgender persons: national population study. Journal of affective disorders, 245, 1126-1134. https://doi.org/10.1016/j.jad.2018.12.011

Claes, L.; Bouman, W.P.; Witcomb, G.; Thurston, M.; Fernandez-Aranda, F.; Arcelus, J. (2015). Non-suicidal self-injury in trans people: Associations with psychological symptoms, victimization, interpersonal functioning, and perceived social support. The Journal of Sexual Medicine, 12(1), 168-179.

Confederación SALUD MENTAL ESPAÑA. (2020). SALUD MENTAL ESPAÑA urge un Plan Nacional de Prevención del Suicidio ante la mayor vulnerabilidad de la salud mental de la población debido a la pandemia. Madrid, España: Confederación SALUD MENTAL ESPAÑA. Recuperado de https://consaludmental.org/

Eisenberg, M.E.; Gower, A.L.; McMorris, B.J.; Rider, G.N.; Shea, G.; Coleman, E. (2017). Risk and protective factors in the lives of transgender/gender nonconforming adolescents. Journal of Adolescent Health, 67(4), 521526. http://dx.doi.org/10.1016/j.jadohealth.2017.04.014

Haas, A.P.; Eliason, M.; Mays, V.M.; Mathy, R.M.; Cochran, S.D.; D'Augelli, A.N.; ... Clayton, P.J. (2011) Suicide and Suicide Risk in Lesbian, Gay, Bisexual, and Transgender Populations: Review and Recommendations. Journal of Homosexuality, 58(1), 10-51. https://doi.org/10.1080/00918369.2011.534038

HBICDA (The Harry Benjamin International Gender Dysphoria Association). (1998). The Standards of care for Gender Identity Disorders Author (Fifth ed.). Mineapolis: HBIGDA.

HBICDA (The Harry Benjamin International Gender Dysphoria Association). (2001). The Standards of care for Gender Identity Disorders (Sixth version) Mineapolis: Author.

Instituto Nacional de Estadística. (2019). Defunciones según la Causa de Muerte. Año 2018. Recuperado de https:// www.ine.es/prensa/edcm_2018.pdf

Lehavot, K.; Simpson, T.L.; Shipherd, J.C. (2016). Factors associated with suicidality among a national sample of transgender veterans. Suicide and Life-Threatening Behavior, 46(5), 507-524. https://doi.org/10.1117/ sltb.12233

Liu, R.T.; Mustanski, B. (2012). Suicidal ideation and self-harm in lesbian, gay, bisexual, and transgender youth. American Journal of Preventive Medicine, 42, 221-228. https://doi.org/10.1016/j.amepre.2011.10.023

Marshall, E.; Claes, L.; Bouman, W.P.; Witcomb. G.L.; Arcelus, J. (2016) Non-suicidal self-injury and suicidality in trans people: A systematic review of the literature. International Review of Psychiatry, 28(1), 58-69. https://doi. org/10.3109/09540261.2015.1073143

Meyer, I.H. (2003). Prejudice, social stress, and mental health in lesbian, gay, and bisexual populations: Conceptual issues and research evidence. Psychological Bulletin, 129(5), 674. 
Modrego Pardo, I.; Gómez Balaguer, M.; Hurtado Murillo, F.; Cuñat Navarro, E.; Solá Izquierdo, E.; Morillas Ariño, C. (2020). Antecedentes de comportamientos autolesivos y autolíticos en población adolescente y adulta joven transexual, atendida en una unidad especializada en identidad de género en España. Endocrinología, Diabetes y Nutrición. https://doi.org/10.1016/j.endinu.2020.04.006

Moody, C.; Smith, N.G. (2013). Suicide protective factors among trans adults. Archives of sexual behavior, 42(5), 739752. https://doi.org/10.1007/s10508-013-0099-8

Nock, M.K.; Borges, G.; Bromet, E.J.; Alonso, J.; Angermeyer, M.; Beautrais, A.; ... De Graaf, R. (2008a). Cross-national prevalence and risk factors for suicidal ideation, plans and attempts. The British Journal of Psychiatry, 192(2), 98-105. https://doi.org/10.1192/bjp.bp.107.040113

Nock, M.K.; Borges, G.; Bromet, E.J.; Cha, C.B.; Kessler, R.C.; Lee, S. (2008b). Suicide and suicidal behavior. Epidemiologic reviews, 30(1), 133-154.

Organización Mundial de la Salud (2 de septiembre de 2019). Suicidio. Recuperado de: https://www.who.int/es/ news-room/fact-sheets/detail/suicide

Perez-Brumer, A.; Day, J.K.; Russell, S.T.; Hatzenbuehler, M.L. (2017) Prevalence and Correlates of Suicidal Ideation Among Transgender Youth in California: Findings From a Representative, Population-Based Sample of High School Students. Journal of the American Academy of Child \& Adolescent Psychiatry, 56(9), 739746. https://doi.org/10.1016/j.jaac.2017.06.010

Taliaferro, L.A.; McMorris, B.J.; Eisenberg, M.E. (2018). Connections that moderate risk of non-suicidal self-injury among transgender and gender non-conforming youth. Psychiatry research, 268, 65-67. https://doi. org/10.1016/j.psychres.2018.06.068

Terada, S.; Matsumoto, Y.; Sato, T.; Okabe, N.; Kishimoto, Y.; Uchitomi, Y. (2011). Suicidal ideation among patients with gender identity disorder. Psychiatry research, 190(1), 159-162.

Testa, R.J.; Michaels, M.S.; Bliss, W.; Rogers, M.L.; Balsam, K.F.; Joiner, T. (2017). Suicidal ideation in transgender people: gender minority stress and interpersonal theory factors. Journal of abnormal psychology, 126(1), 125.

Tomicic, A.; Gálvez, C.; Quiroz, C.; Martínez, C.; Fontbona, J.; Rodríguez, J.; Aguayo, F.; Rosenbaum, C.; Leyton, F.; Lagazzi, I. (2016). Suicidio en poblaciones lesbiana, gay, bisexual y trans: revisión sistemática de una década de investigación (2004-2014). Revista médica de Chile, 144(6), 723-733.

Wolford-Clevenger, C.; Frantell, K.; Smith, P.N.; Flores, L.Y.; Stuart, G.L. (2018) Correlates of suicide ideation and behaviors among transgender people: A systematic review guided by ideation-to-action theory. Clinical Psychology Review, 63, 93-105. https://doi.org/10.1016/j.cpr.2018.06.009 